Article

\title{
Ferulic Acid Improves Depressive-Like Behavior in Prenatally-Stressed Offspring Rats via Anti-Inflammatory Activity and HPA Axis
}

\author{
Xingxing Zheng ${ }^{1,2, \dagger}$, Ying Cheng ${ }^{1,2, \dagger}$, Yiwei Chen ${ }^{1,2}$, Yisong Yue ${ }^{1,2}$, Yingchun $\mathrm{Li}^{1,2}$, \\ Sizhe Xia ${ }^{1,2}$, Yang Li ${ }^{1,2}$, Huanhuan Deng ${ }^{1,2}$, Junli Zhang ${ }^{1,2}$ and Yanjun Cao ${ }^{1,2, *}$ \\ 1 Key Laboratory of Resource Biology and Biotechnology in Western China (Northwest University), Ministry \\ of Education, Xi'an 86-710069, China; zheng@stumail.nwu.edu.cn (X.Z.); 15829758592@163.com (Y.C.); \\ 2016113142@stumail.nwu.edu.cn (Y.C.); yueyeson123@gmail.com (Y.Y.); \\ 201831945@stumail.nwu.edu.cn (Y.L.); xiasizhe@stumail.nwu.edu.cn (S.X.); \\ 2017113115@stumail.nwu.edu.cn (Y.L.); m17782526319@163.com (H.D.); 15191905972@163.com (J.Z.) \\ 2 Shaanxi Province Biomedicine Key Laboratory, College of Life Sciences, Northwest University, \\ Xi'an 710069, China \\ * Correspondence: cao2014@nwu.edu.cn \\ + These authors contributed equally to this paper.
}

Received: 10 December 2018; Accepted: 18 January 2019; Published: 24 January 2019

\begin{abstract}
Prenatal stress (PS) can increase the risk of nervous, endocrine and metabolic diseases, and immune dysfunction. Ferulic acid (FA) is a dietary phenolic acid that has pharmacological properties, including potent anti-inflammatory action. We used male, prenatally-stressed offspring rats to investigate the anti-depressive-like effects and possible anti-inflammatory mechanism of FA. We determined the animal behaviors, and the mRNA expression and concentration of inflammatory cytokines, and HPA axis. In addition, we assessed the modulation of hippocampal nuclear factor- $\mathrm{kB}(\mathrm{NF}-\mathrm{kB})$ activation, neuronal nitric oxide synthase (nNOS) and glucocorticoid receptors (GR) expression via western blotting and immunohistochemistry. Administration of FA $(12.5,25$, and $50 \mathrm{mg} / \mathrm{kg} /$ day, i.g.) for 28 days markedly increased sucrose intake, and decreased immobility time and total number of crossings, center crossings, rearing, and grooming in the male PS offspring. FA significantly reduced IL-6, IL- $1 \beta$, and TNF- $\alpha$ concentration and increased IL-10 concentration in male, prenatally-stressed offspring, stimulated by the NF- $\mathrm{kB}$ pathway. In addition, FA inhibited interleukin-6 (IL-6), interleukin-1 $\beta$ (IL-1 $\beta)$, and tumor necrosis factor- $\alpha$ (TNF- $\alpha)$, and increased interleukin-10 (IL-10) mRNA and protein expression. Furthermore, FA markedly decreased the serum adrenocorticotropin $(\mathrm{ACTH})$ and corticosterone concentration by the increase of GR protein expression. Taken together, this study revealed that FA has anti-depressive-like effects in male, prenatally-stressed offspring, partially due to its anti-inflammatory activity and hypothalamic-pituitary-adrenal (HPA) axis.
\end{abstract}

Keywords: Ferulic Acid; Prenatal stress; Depression; NF-kB; glucocorticord receptor; Inflammatory cytokines

\section{Introduction}

Prenatal stress (PS), which refers to stress during pregnancy, has been reported to exert a wide variety of negative emotional and behavioral effects on both human and animal offspring, including depression, anxiety, attention deficit hyperactivity disorder, and especially learning and memory deficits [1-3]. PS increases reactivity of the hypothalamic-pituitary-adrenal (HPA) axis in rats [4,5], 
which may affect immune responses. Immune cells express receptors for a variety of hormones [6] and glucocorticoids (GCs) can affect the development and function of the immune system $[7,8]$.

Depression is a common mental disorder with symptoms including loss of interest or pleasure (anhedonia), a depressed mood, decreased energy, disturbed sleep or appetite, poor concentration and feelings of guilt or low self-worth [9]. There is no direct neural connection between the mother and fetus, the alterations of neuronal morphology induced by maternal stress are produced by the action of hormones at crucial periods of development. Prolonged GC exposure of the mother can reduce the number of hippocampal neurons of the offspring [10]. The hippocampus is particularly vulnerable to the effects of GCs because of the highest density of glucocorticoid receptor (GR) within the brain, which is intrinsically linked to both learning and memory and emotion. It has been reported that PS increases the HPA axis reactivity and circulating levels of the stress hormones GCs, and decreases the glucocorticoids receptor (GR) protein expression [11]. Therefore, reduction in serum corticosterone level may contribute to protecting the hippocampus neurons and improve depressive behavior. For the immature immune system of intrauterine fetus, elevated GCs can reduce the ability of self-protection, especially in terms of cellular immunity [6]. GCs can influence the process of maturation and growth of leukocyte, and regulate many cytokines [8,12]. Study has shown that inflammation plays a pivotal role in the pathogenesis of depression [9]. Animal models of depressive disorders have shown an increase in pro-inflammatory markers, such as interleukin-6 (IL-6), interleukin-1 $\beta$ (IL-1 $\beta$ ) and tumor necrosis factor alpha (TNF- $\alpha$ ). In addition, IL-1 $\beta$, IL-6, and TNF- $\alpha$ mRNA expression are increased in the cortex, hypothalamus, and hippocampus of stressed rats $[13,14]$. Nuclear factor (NF)- $\mathrm{\kappa B}$ is a pro-inflammatory transcription factor and a major mediator of inflammatory pathways, and regulated many cytokines, such as TNF- $\alpha$, IL-6, IL-1, and IL-10. Many antidepressants have been shown to suppress NF-kB activation and pro-inflammatory transcription [15-18].

In recent years, Chinese traditional medicine has begun to focus more on the treatment of depression. Ferulic acid (FA) (4-hydroxy-3-methoxycinnamic acid), is a phenolic acid which is present in many plants such as Ferula teterrima Kar. Et Kir., Angelica sinensis, Cimicifuga foetida L., and Ligusticum chuanxiong Hort. It has a variety of biological effects including anti-inflammatory, anti-epileptogenic, anticancer and antioxidant activities $[19,20]$. In particular, a large number of animal experiments also show that FA can reverse memory loss in mice caused by inflammation, elevate the carbonyl protein level and reduce nerve cell injury [20-23]. In our previous studies, we focused on the negative effect of PS on the offspring and the specific mechanism, including the impaired GR and increased HPA axis reactivity. Taken together, these studies suggest that FA improves the depression induced by stress, but the effect and underlying mechanisms remain unclear. Therefore, these findings compelled us to explore whether FA had an effect on improving depression induced by PS in offspring.

The major objective of this study was to investigate the possible anti-depressive and anti-inflammatory effects of FA in prenatally-stressed offspring, and its possible mechanism. After 28 days of FA treatment in prenatally-stressed offspring rats, we measured the behavioral tests, including the sucrose preference test, forced swimming test (FST) and open field test (OFT). Then we detected the concentration and the expression of mRNA of TNF- $\alpha$, IL-6, IL-10 and IL-1 $\beta$ in the hippocampus. We also measured the serum adrenocorticotropin (ACTH) and corticosterone levels, and evaluated the protein expression of NF-kB and GR in the hippocampus.

\section{Results}

\subsection{Effects of FA on Sucrose Preference Test}

There was a significant reduction in the percentage of sucrose consumed in PS rats when compared with the control group respectively ( $p<0.05$; Figure 1). Moreover, when FA-L, FA-M, FA-H, or fluoxetine were administrated for 28 days, there was a significant increase in sucrose preference compared with the PS group $(p<0.05$; Figure 1$)$. 
A

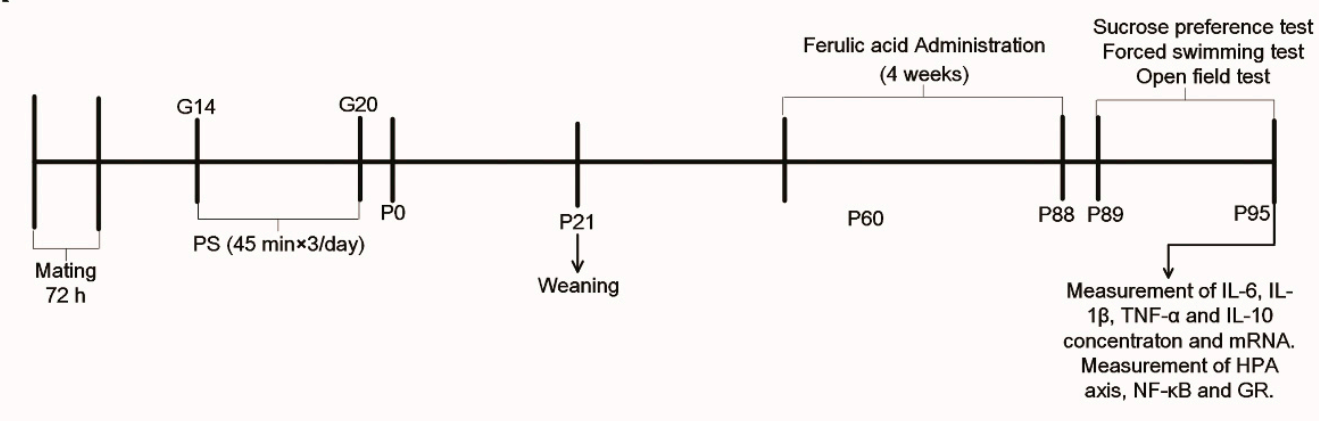

B<smiles>COc1cc(/C=C/C(=O)O)ccc1O</smiles>

Ferulic acid
C

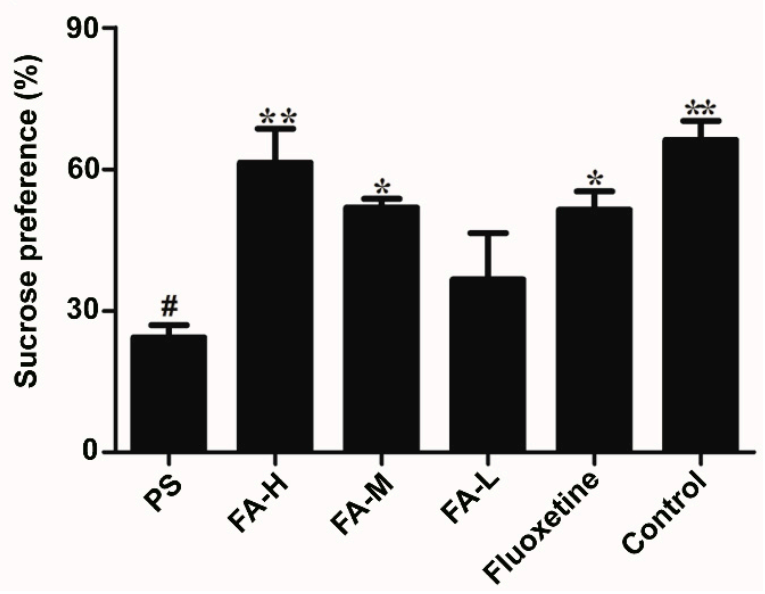

Figure 1. Chemical structure and effects of ferulic acid (FA) on the sucrose preference in offspring rats exposed to prenatal stress. (A) Timeline showing a summary of the experimental design. G: gestational age; P: postnatal age (days). PS: prenatal stress; (B) Chemical structure of FA; (C) Effects of FA on the sucrose preference. The percentage of sucrose consumed at sucrose concentrations of $2 \%$. Values represent means $\pm \operatorname{SEM}(n=8) .{ }^{*} p<0.05$ and ${ }^{* *} p<0.01$ compared with PS group, ${ }^{\#} p<0.05$ compared with Control group. FA-L: FA at low $12.5 \mathrm{mg} / \mathrm{kg} /$ day, FA-M: FA at medium $25 \mathrm{mg} / \mathrm{kg} /$ day, FA-H: FA at high $50 \mathrm{mg} / \mathrm{kg} /$ day.

\subsection{Effects of FA on Immobility in the Forced Swim Test}

The result of FST is presented in Figure 2. After PS treatment, immobility time $(p<0.05$, Figure 2A) and the immobility time percentage $(p<0.05$, Figure $2 \mathrm{~B})$ in the male offspring were significantly increased compared with control group respectively. Meanwhile, following the administration of FA-L, FA-M, FA-H or fluoxetine for 28 days, the immobility time ( $p<0.05$, Figure $2 \mathrm{~A})$ and the immobility time percentage $(p<0.05$, Figure $2 \mathrm{~B})$ were remarkably decreased in the male offspring. 
A

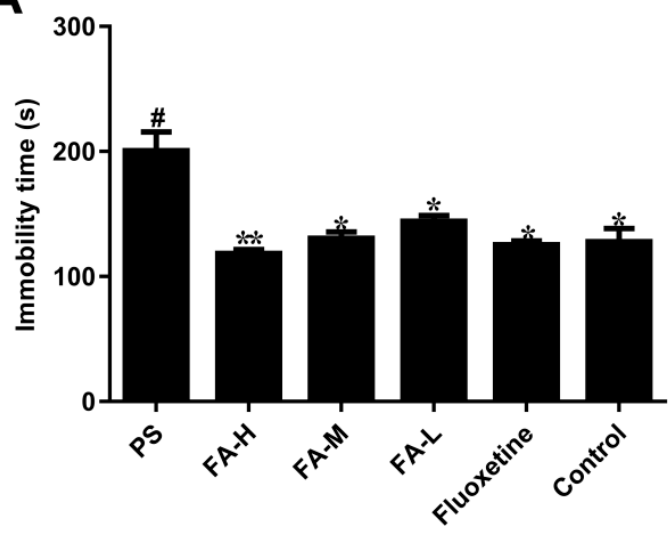

B

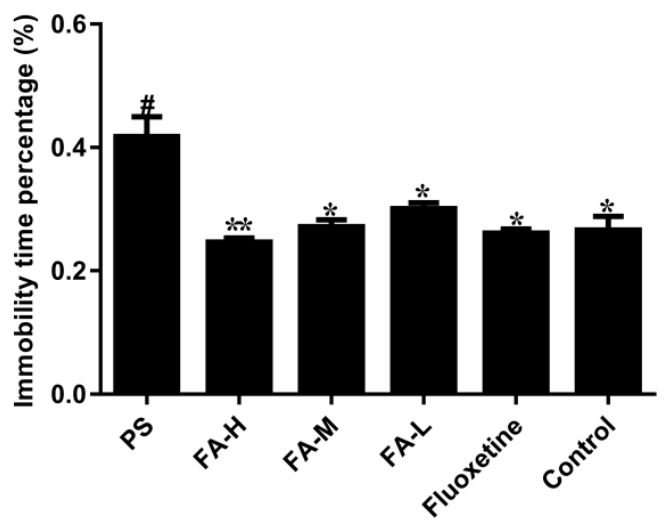

Figure 2. Effects of ferulic acid (FA) on the forced swimming test of offspring rats exposed to prenatal stress. (A) Immobility time in forced swimming test. (B) Immobility time percentage in forced swimming test. Values represent means $\pm \operatorname{SEM}(n=8) .{ }^{*} p<0.05$ and ${ }^{* *} p<0.01$ compared with PS group, ${ }^{\#} p<0.05$ compared with Control group. FA-L: FA at low $12.5 \mathrm{mg} / \mathrm{kg} /$ day, FA-M: FA at medium $25 \mathrm{mg} / \mathrm{kg} /$ day, FA-H: FA at high $50 \mathrm{mg} / \mathrm{kg} /$ day.

\subsection{Effects of FA on Open Field Test}

As shown in Figure 3, there was a reduction in the number of center crossings $(p<0.05)$, total crossings $(p<0.05)$, rearing $(p<0.05)$, and grooming $(p<0.05)$ in PS offspring rats when compared with control rats. Treatment with FA significantly increased the PS-induced reduction $(p<0.05)$.

A
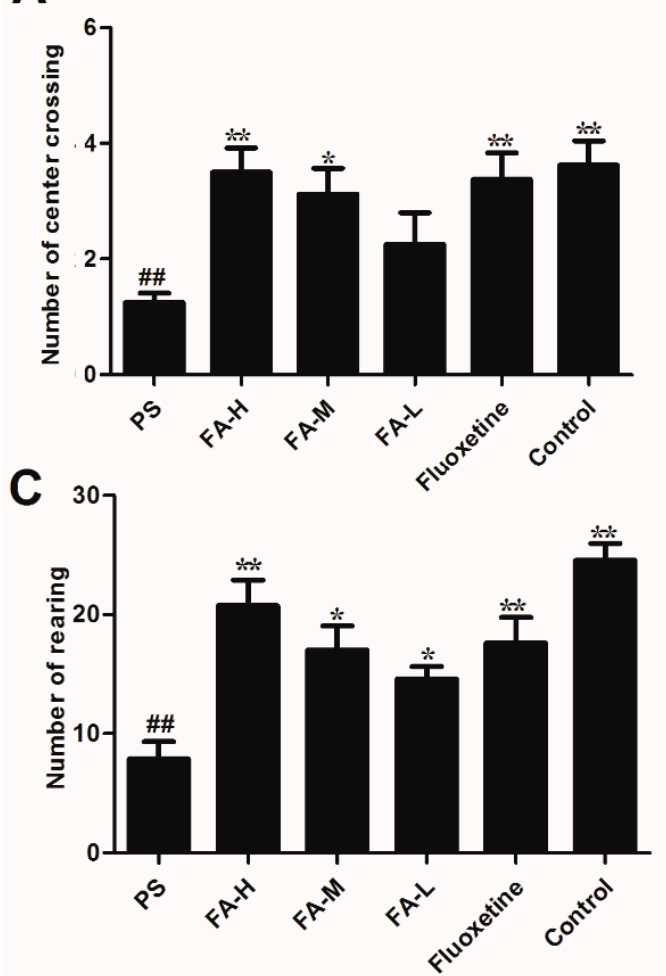

B
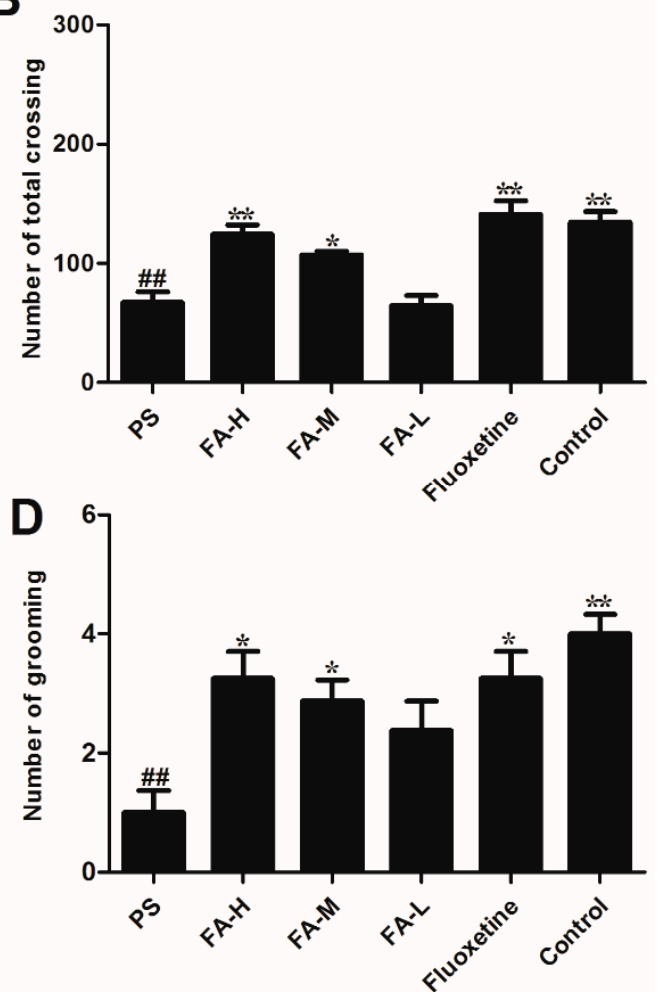

Figure 3. Effects of ferulic acid (FA) on the open field test of offspring rats exposed to prenatal stress. (A) The number of total crossings. (B) The number of center crossings. (C) The number of rearing. (D) The number of grooming. Values represent means $\pm \operatorname{SEM}(n=8) .{ }^{*} p<0.05$ and ${ }^{* *} p<0.01$ compared with PS group, ${ }^{\#} p<0.01$ compared with Control group. FA-L: FA at low $12.5 \mathrm{mg} / \mathrm{kg} /$ day, FA-M: FA at medium $25 \mathrm{mg} / \mathrm{kg} /$ day, FA-H: FA at high $50 \mathrm{mg} / \mathrm{kg} /$ day; Flx: fluoxetine. 


\subsection{Effects of FA on ACTH and Corticosterone Levels}

To test if the protective role of FA in depressive-like behavior induced by PS is achieved by reducing the levels of serum ACTH and corticosterone, we assessed the effects of FA on serum ACTH and corticosterone levels in offspring rats exposed to PS (Figure 4). There was a significant interaction $(p<0.01)$, with effects for PS offspring tars $(p<0.01)$ and difference in FA treatment $(p<0.05)$ with serum ACTH and corticosterone levels. FA significantly reversed the elevated serum ACTH and corticosterone levels in offspring rats exposed to PS. Interestingly, fluoxetine treatment markedly prevented PS-induced offspring changes higher than those in the control group in serum ACTH and corticosterone levels. These results indicate that FA improves the depressive-like behavior by affecting the levels of serum ACTH and corticosterone.
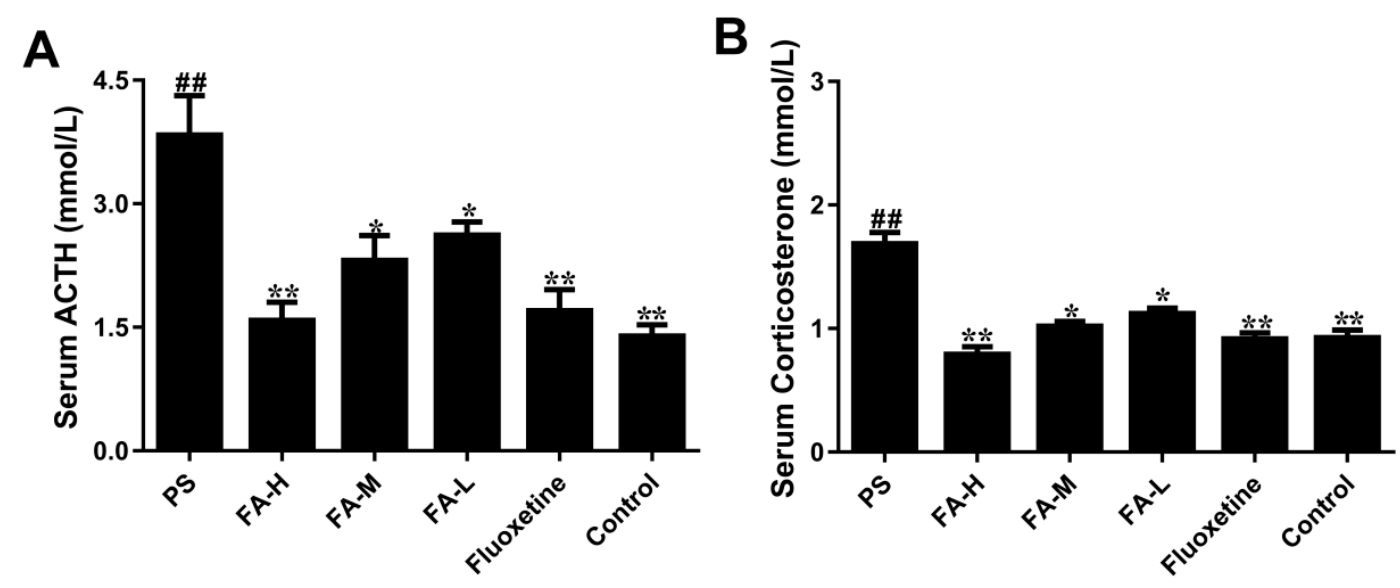

Figure 4. Effects of ferulic acid (FA) on the serum adrenocorticotropin (ACTH) and corticosterone levels of offspring rats exposed to prenatal stress. (A) Serum ACTH levels. (B) Serum corticosterone levels. Values represent means $\pm \operatorname{SEM}(n=8) .{ }^{*} p<0.05$ and ${ }^{* *} p<0.01$ compared with PS group, \#\# $p<0.01$ compared with Control group. FA-L: FA at low $12.5 \mathrm{mg} / \mathrm{kg} /$ day, FA-M: FA at medium 25 $\mathrm{mg} / \mathrm{kg}$ /day, FA-H: FA at high $50 \mathrm{mg} / \mathrm{kg} /$ day.

\subsection{Effects of FA on Nissl Staining in the Hippocampus of Offspring}

Typical neuropathological changes, including neuron atrophy and nucleus shrinkage, were observed by Nissl staining in the CA3 of hippocampus in PS group, and these neuronal damages were ameliorated after the treatment of FA $(p<0.05$, Figure 5$)$. 
A
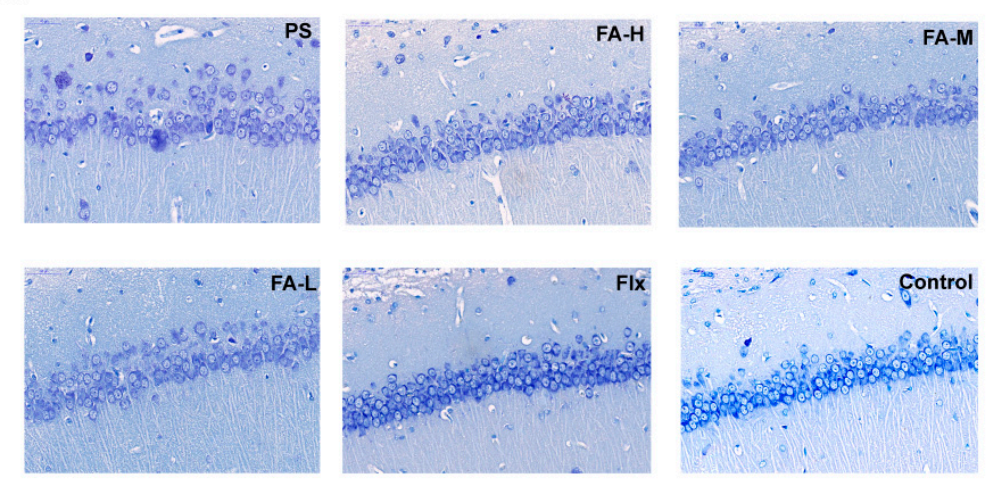

B

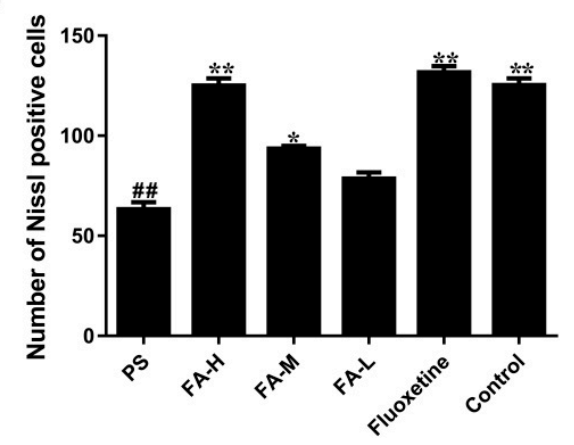

Figure 5. Effect of ferulic acid (FA) on hippocampus CA3 region neuron density in offspring rats exposed to prenatal stress. (A) Nissl staining of hippocampus CA3 region sections (magnification $400 \times$, Scale bar $=50 \mu \mathrm{m}$ ), and (B) quantification of Nissl bodies in the hippocampus CA3 region. Data were expressed as mean \pm SEM. $n=3$ in each group, indicating at least three independent experiments in each animal. ${ }^{*} p<0.05$ and ${ }^{* *} p<0.01$ compared with PS group, ${ }^{\# \#} p<0.01$ compared with Control group. FA-L: FA at low $12.5 \mathrm{mg} / \mathrm{kg} /$ day, FA-M: FA at medium $25 \mathrm{mg} / \mathrm{kg} /$ day, FA-H: FA at high $50 \mathrm{mg} / \mathrm{kg} /$ day; Flx: fluoxetine.

\subsection{Effects of FA on Neuronal NOS-Positive Expression in the Hippocampus of Offspring}

A high expression of neuronal nitric oxide synthase (nNOS) in the PS group was detected by immunohistochemical analysis ( $p<0.05$ vs. control group) (Figure 6), which indicated that PS induced inflammation in the CA3 region of the hippocampus. The integrated optical density (IOD) of nNOS was decreased in fluoxetine group ( $p<0.05$ vs. PS group), and markedly decreased in FA groups in a concentration-dependent manner ( $p<0.05$ vs. PS group; Figure 6). 
A
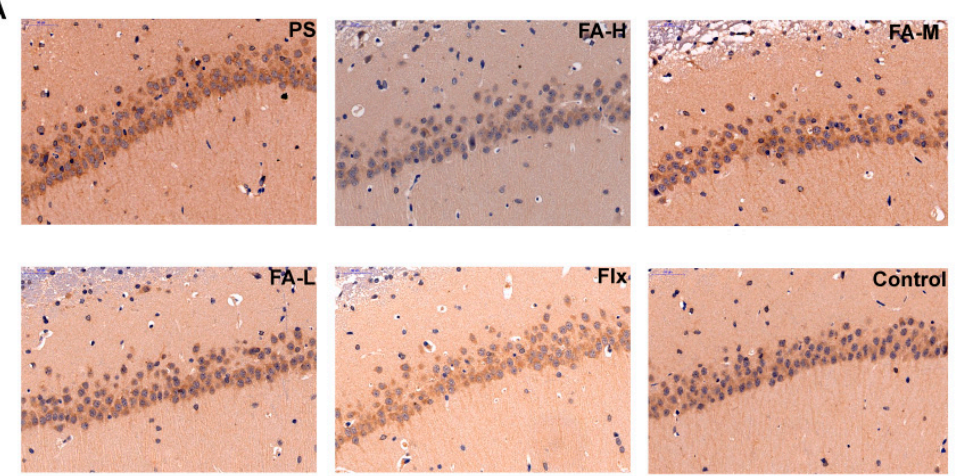

B

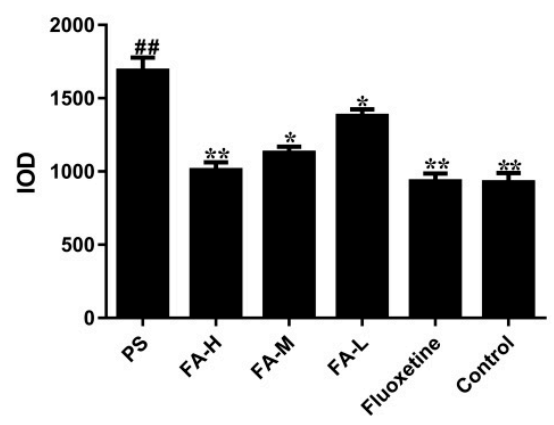

Figure 6. Effects of ferulic acid (FA) on the neuronal nitric oxide synthase (nNOS)-positive expression in hippocampal CA3 region of offspring rats exposed to prenatal stress. (A) nNOS expression was examined by immunohistochemistry (magnification $400 \times$, Scale bar $=50 \mu \mathrm{m}$ ), and (B) Bar graph showing semi-quantitative analysis of nNOS via quantified integrated optical density (IOD). Values represent means $\pm \operatorname{SEM}(n=3) .{ }^{*} p<0.05$ and ${ }^{* *} p<0.01$ compared with PS group, ${ }^{\# \#} p<0.01$ compared with Control group. FA-L: FA at low $12.5 \mathrm{mg} / \mathrm{kg} /$ day, FA-M: FA at medium $25 \mathrm{mg} / \mathrm{kg} /$ day, FA-H: FA at high $50 \mathrm{mg} / \mathrm{kg} /$ day; Flx: fluoxetine.

\subsection{Effects of FA on Cytokine Production}

As shown in Figure 7, the levels of TNF- $\alpha$, IL-1 $\beta$ and IL-6 in hippocampus were markedly increased in PS offspring rats when compared to control group $(p<0.05)$, while IL-10 level was significantly decreased after expose to PS. FA treatment can significantly reduce the TNF- $\alpha$, IL- $1 \beta$ and IL-6 levels, and increase the IL-10 levels in offspring rats expose to PS $(p<0.05)$. 
A

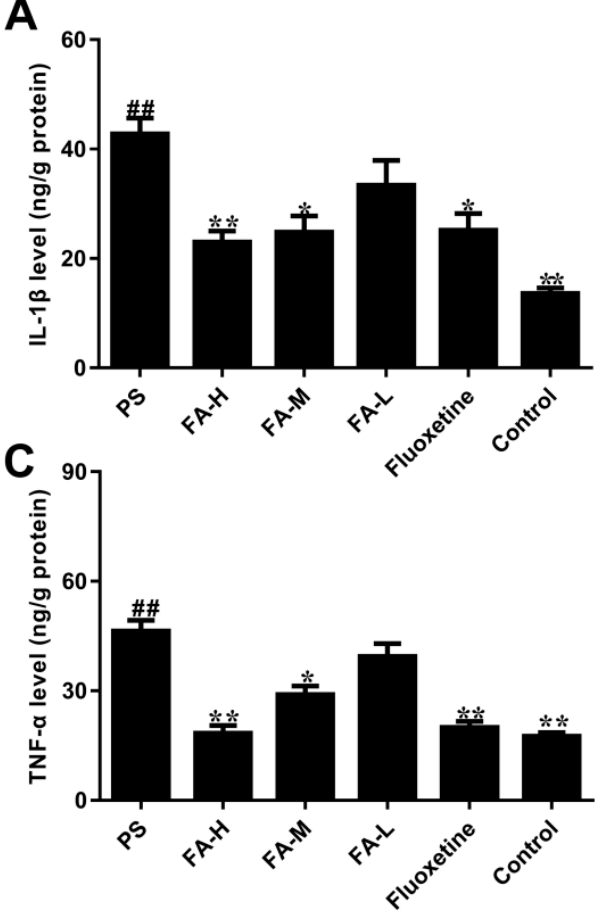

B

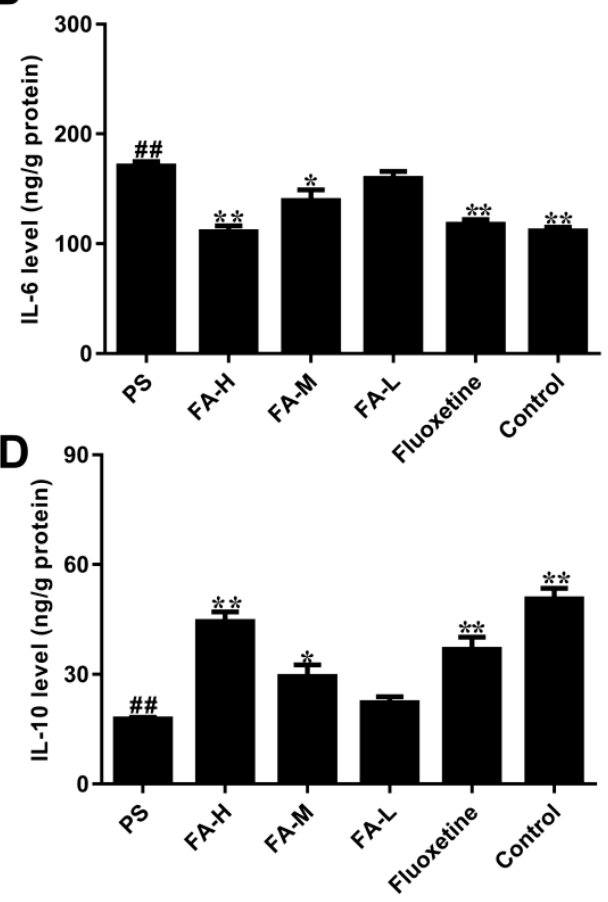

Figure 7. Effects of ferulic acid (FA) on the cytokine concentration in the hippocampus of offspring rats exposed to prenatal stress. (A) IL- $1 \beta$ concentration in the hippocampus. (B) IL-6 concentration in the hippocampus. (C) TNF- $\alpha$ concentration in the hippocampus. (D) IL-10 concentration in the hippocampus. Values represent means \pm SEM $(n=8) .{ }^{*} p<0.05$ and ${ }^{* *} p<0.01$ compared with PS group, ${ }^{\# \#} p<0.01$ compared with Control group. FA-L: FA at low $12.5 \mathrm{mg} / \mathrm{kg} /$ day, FA-M: FA at medium $25 \mathrm{mg} / \mathrm{kg} /$ day, FA-H: FA at high $50 \mathrm{mg} / \mathrm{kg} /$ day.

\subsection{Effects of FA on Cytokine mRNA Expression}

Consistent with the ELISA results, PS resulted in a significant increase in mRNA expression of TNF- $\alpha, I L-1 \beta$, and $I L-6$ in the hippocampus in offspring rats when compared with the control rats $(p<0.05)$. In addition, $I L-10$ mRNA expression was significantly increased after expose to PS. FA treatment significantly inhibited the PS-induced increase in mRNA expression of TNF- $\alpha, I L-1 \beta$ and $I L-6$, and caused a reduction of IL-10 mRNA expression $(p<0.05$; Figure 8$)$. 

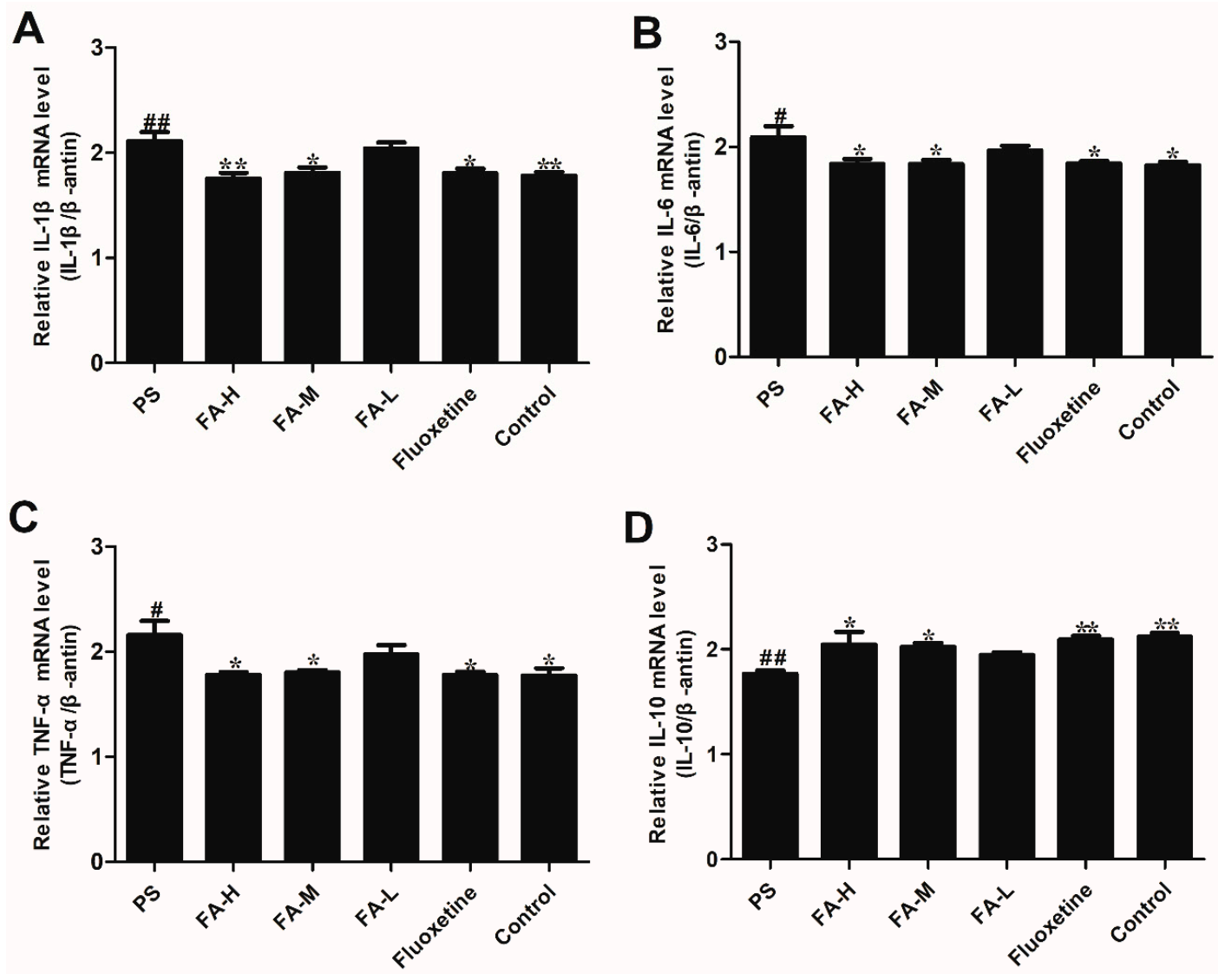

Figure 8. Effects of ferulic acid (FA) on the cytokine mRNA expression in the hippocampus of offspring rats exposed to prenatal stress. Results of (A) $I L-1 \beta$ mRNA in hippocampus of the offspring. (B) $I L-6$ mRNA in in the hippocampus. (C) TNF- $\alpha$ mRNA in the hippocampus. (D) $I L-10$ mRNA in the hippocampus were quantified by real-time PCR. Values represent means $\pm \operatorname{SEM}(n=3) .{ }^{*} p<0.05$ and ** $p<0.01$ compared with PS group, ${ }^{\#} p<0.05$ and ${ }^{\# \#} p<0.01$ compared with Control group. FA-L: FA at low $12.5 \mathrm{mg} / \mathrm{kg} /$ day, FA-M: FA at medium $25 \mathrm{mg} / \mathrm{kg} /$ day, FA-H: FA at high $50 \mathrm{mg} / \mathrm{kg} /$ day.

\subsection{Effects of FA on NF- $\kappa B$ Activation and GR Expression}

NF- $\mathrm{kB}$ is an important upstream modulator of pro-inflammatory cytokines. Thus, we determined whether the FA-induced attenuation in cytokine expression occurred due to a blockade of NF- $\mathrm{kB}$ activity. As shown in Figure 9, the chronic treatment of FA markedly suppressed the phosphorylation of NF-KB in the hippocampus in male PS offspring rats. 
A

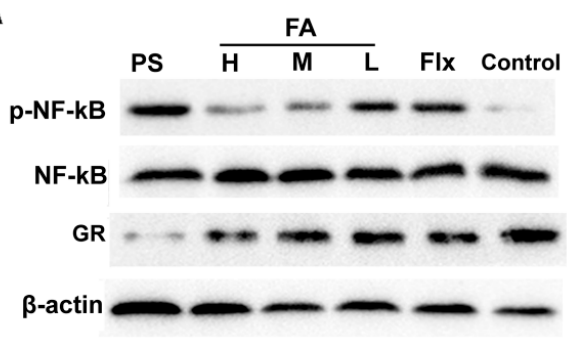

C

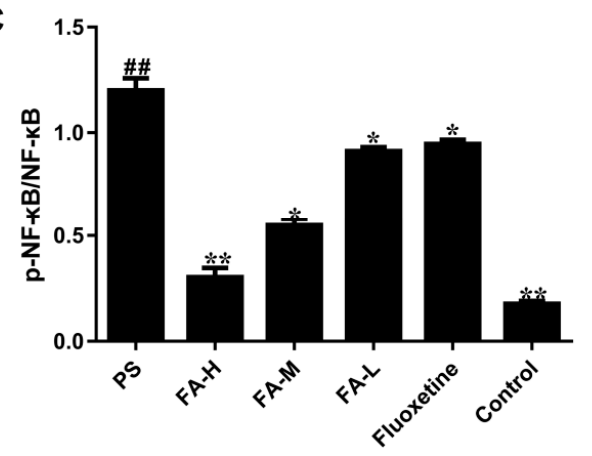

E

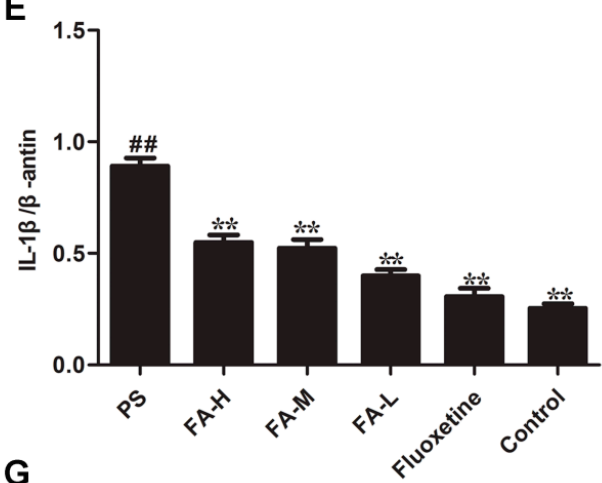

G

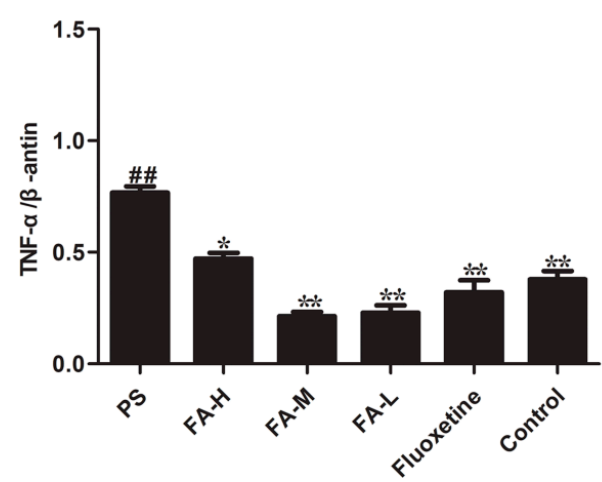

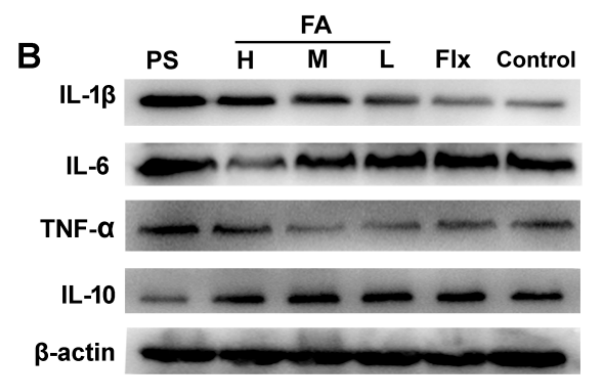

D
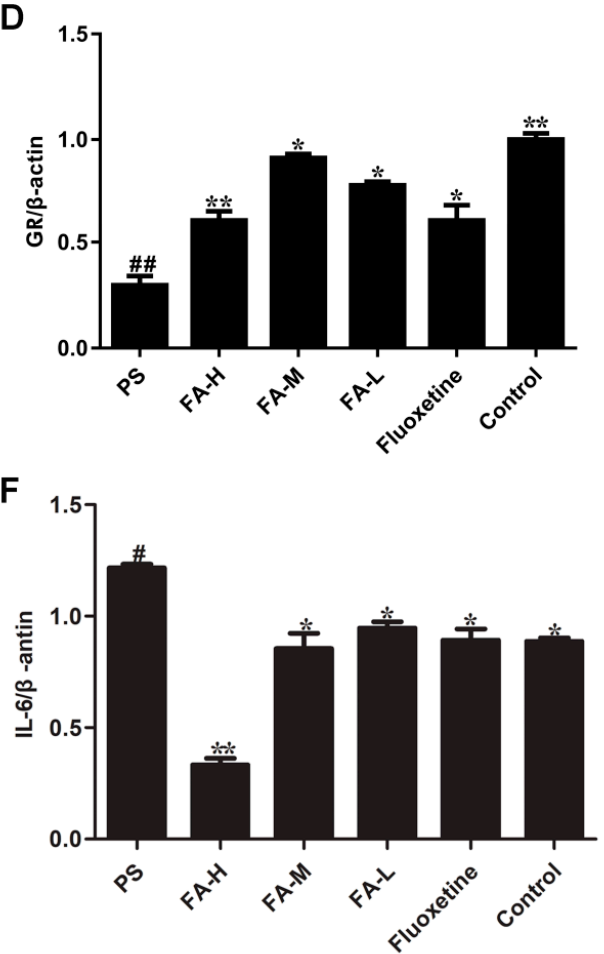

H

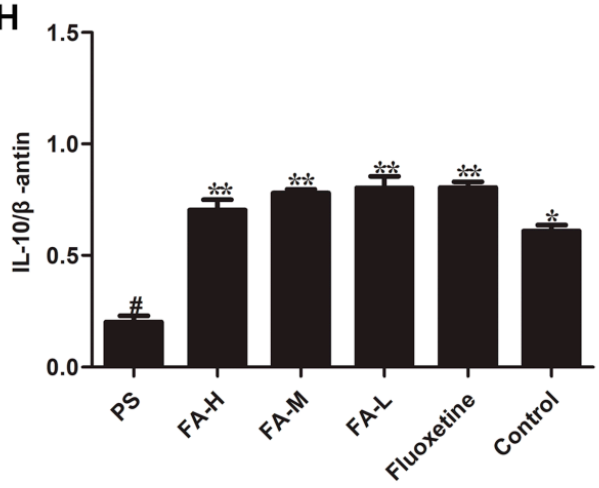

Figure 9. Effects of ferulic acid (FA) on the NF- $\mathrm{kB}$ activation, GR and IL family members expression in the hippocampus of offspring rats exposed to prenatal stress. (A) Bands of p-NF- $k B / N F-k B$ and GR in the hippocampus. (B) Bands of IL family in the hippocampus. (C) Bands of p-NF-kB were quantified based on densitometric analysis and then normalization to NF- $\mathrm{KB}$. (D) Bands of GR were quantified based on densitometric analysis and then normalization to $\beta$-actin protein levels. Bands of (E) IL-1 $\beta$, (F) IL-6, (G) TNF- $\alpha$ and (H) IL-10 were quantified based on densitometric analysis and then normalization to $\beta$-actin protein levels. Values represent means $\pm \operatorname{SEM}(n=3) .{ }^{*} p<0.05$ and ${ }^{* *} p<0.01$ compared with PS group, ${ }^{\#} p<0.01$ and ${ }^{\# \#} p<0.01$ compared with Control group. FA-L: FA at low $12.5 \mathrm{mg} / \mathrm{kg} /$ day, FA-M: FA at medium $25 \mathrm{mg} / \mathrm{kg} /$ day, FA-H: FA at high $50 \mathrm{mg} / \mathrm{kg} /$ day. 
PS significantly decreased the expression of GR in the hippocampus of offspring rats (Figure 9) compared with the control group $(p<0.05)$. However, FA treatment significantly increased the hippocampal GR protein expression in offspring rats expose to PS $(p<0.05)$. Moreover, effects of FA were similar to fluoxetine group.

In order to explore the influence of FA on IL family protein expression in rat offspring, we measured TNF- $\alpha$, IL-1 $\beta$, IL- 6 and IL-10 protein expression hippocampus of offspring rats. The results revealed that FA significantly decreased TNF- $\alpha$, IL-1 $\beta$ and IL-6 protein expression and significantly increased IL-10 protein expression in the hippocampus of offspring rats compared to the PS group $(p<0.05$, Figure 9).

\section{Discussion}

This study sought to determine the effect of FA on inflammatory mediators and depressive-like behaviors in male offspring rats exposed to PS. We have found that PS-induced anxiety- and depressive-like behaviors were associated with increase in hippocampal inflammatory mediators and HPA axis reactivity. Furthermore, FA administration attenuated the increase in the pro-inflammatory cytokines, IL-1 $\beta$, IL-6, TNF- $\alpha$, and decreased IL-10 and depressive-like behavior in PS offspring rats. PS upregulated the hippocampal $I L-1 \beta, I L-6, T N F-\alpha$, and downregulated the IL-10 mRNA expression in male offspring rats, which were attenuated by chronic FA treatment. Meanwhile, PS resulted in a marked increase in hippocampal expression of NF- $\mathrm{kB}$ and $\mathrm{nNOS}$, which was also attenuated by chronic FA treatment. We also found that FA significantly decreased the ACTH and corticosterone levels in the hippocampus of male PS offspring rats, associated with increased GR expression. These results suggest that the effects of FA may be dependent on its antidepressant and anti-inflammatory effects.

It is well known that the hippocampus is involved in emotional regulation. Many studies have shown that inflammation in this region may lead to depression and anxiety $[5,24,25]$. Anhedonia has been defined as a decrease in responsiveness to rewards reflected by a reduced intake of palatable sweet solutions, which is a core symptom of human depression. The sucrose preference test has been known to an index of anhedonia-like behavioral alteration $[8,26]$. In our study, we found that the PS-induced reduction in sucrose preference was significantly reversed by chronic FA treatment, suggesting an anti-depressive-like action of FA.

The early life environment is crucial for individual development. Gestational stress increases maternal circulating GCs. In addition, stress hormones can cross the placental barrier and potentially result in permanent adverse short- and long-term neuroanatomical, biological, behavioral and immune modifications in the offspring [27,28]. GCs can influence the process of maturation and growth of leukocyte, and regulate cytokines include TNF- $\alpha$, IL-4 and IL-10 secreted by Th1 cells or Th2 cells which modulate cell immune responses and humoral immune responses [8,12]. It has been reported that many cytokines play an important role in stress-related inflammatory disorders and neurological diseases [13]. In IL-6-deficient mice, the results have shown that this cytokine can affect emotional related behaviors, especially anxiety-related behaviors [7]. If cytokines in the brain are dysregulated, they may cause corresponding pathologic changes, including inflammatory, oxidative, and nitrosative molecules and affect neural homeostasis [25]. Increases in serum pro- and anti-inflammatory cytokines have been observed in patients with depression, such as IL-1 $\beta$, IL-6, and TNF- $\alpha$, and IL-10, respectively. PS induces alterations in the HPA axis in rats, which can alter immune reactivity [11]. In our study, PS increased the mRNA expression of $I L-1 \beta, I L-6$, and TNF- $\alpha$ mRNA expression in the hippocampus of male offspring, and decreased IL-10 mRNA expression, in line with previous studies [7,25]. Chronic treatment with FA dramatically attenuated the PS-induced elevation of pro-inflammatory cytokines. Taken together, these data suggest that the antidepressant-like effects of FA are associated with suppression of pro-inflammatory cytokines.

PS induces the HPA axis reactivity and increases circulating concentration of the stress hormone GCs, and impairs the GR protein in the offspring rats. The hippocampus highly expresses GR, and is particularly vulnerable to GCs manipulations, especially in early life. Prolonged GCs may cause 
increased activation of excitatory amino acid receptors, including ionotropic glutamate receptors and metabotropic glutamate receptors, and unregulated increases in intracellular $\mathrm{Ca}^{2+}$ concentrations, and consequently, increased generation of oxidants, oxidative and inflammatory damage in males [10]. In our study, the hippocampal nNOS expression in offspring rats was measured and found that the nNOS expression in offspring increased significantly in the PS group. According to these results, FA treatment significantly decreased the PS-induced nNOS expression in the hippocampus of male offspring rats. Corticosterone can cross placenta to affect fetal development and induces overproduction of nNOS in PS offspring rats. The overproduction of nNOS in hippocampal neurons therefore indicates that the neurons in the PS group are in a higher oxidative environment, which may gradually cause neuronal injuries. The expression of nNOS increases significantly in the hippocampus of offspring rats exposed to PS rats, indicating that this CA3 region is under higher inflammation and oxidant stress [29]. We also found the neurons decrease significantly in this region. Evidence could help to explain why pyramidal neurons of CA3 are more vulnerable to PS; granule cells of the DG express a high density of GR [11] and these neurons project heavily to CA3 pyramidal cells via mossy fiber output, which might make the neurons of CA3 and DG more vulnerable to the stress levels of GCs. CA3 pyramidal neurons therefore may be particularly vulnerable to PS. Interestingly, in the present study, we found FA decreased serum ACTH and corticosterone levels in offspring rats exposed to PS, suggesting that FA improves depressive-like behavior through the regulation on HPA axis and GR.

NF- $\mathrm{kB}$ transcriptionally regulates many cellular genes implicated in early immune, acute phase, and inflammatory responses, inducible NO synthase (iNOS), and pro-inflammatory cytokine. Inhibition of NF- $\mathrm{kB}$ activation can suppress the expression of pro-inflammatory cytokines, such as IL-1 $\beta$, IL-6 and TNF- $\alpha$ [30]. In our study, we demonstrated that FA inhibited the PS-induced activation of NF-KB in the hippocampus of offspring rats, which indicates that the FA-induced inhibition of pro-inflammatory gene expression may dependent on modulation of the NF- $\mathrm{KB}$ signaling pathway. FA has shown anti-depressant effects in the tail suspension test in acute depression [31,32], and attenuated chronic neuro inflammation in Alzheimer's disease [33]. In our study, FA attenuated depressive-like behavior in the male offspring rats exposed PS. The effect is mediated by the NF- $\mathrm{kB}$ signaling pathway and GR expression. The results from our present study indicate that FA could be an effective therapeutic treatment in preventing the development of stress-induced, depressive-like behavior in adolescence.

\section{Materials and Methods}

\subsection{Animals and Procedures}

All animal procedures were carried out in line with the National Institutes of Health Guide for the Care and Use of Laboratory Animals and were ratified by the Institutional Animals Care and Use Committee. All efforts were made to minimize suffering and the number of rats used for the experiments. Male Sprague-Dawley (SD) rats weighing 280-350 g and female SD rats weighing 230-250 g were used for our research. The SD rats were purchased from the experimental animal center of Medical College in Xi'an JiaoTong University. All animals were housed in an animal room with controlled humidity $(55 \pm 5 \%)$ and temperature $\left(25 \pm 2{ }^{\circ} \mathrm{C}\right)$ with a 12-h-light/dark cycle (light on from 07:30 to 19:30) with free access to drinking water and food. Virgin female rats and adult male rats (3:1) were housed in one cage for mating; a vaginal smear was tested before 8:00 on the following morning. The day that the smear was sperm positive was defined as embryonic day 0 . Thereafter, pregnant rats were housed separately.

Pregnant rats were separately exposed to restraint stress on days 14-20 of pregnancy three times daily for $45 \mathrm{~min}$, as described previously [34]. To prevent the adaptation of animals to the daily procedure, restraint periods were shifted randomly within certain time periods (08:00 A.M.-11:00 A.M., 11:00 A.M.-2:00 P.M., and 4:00 P.M.-7:00 P.M.). A transparent plastic tube (6 $\mathrm{cm}$ in diameter) 
with breathing holes was used as the restraint device. The length of the plastic tube was suited for the size of the animals. Control pregnant rats were not disturbed. All offspring rats were weaned on postnatal day 21, and then separated and housed at no more than four offspring per cage. At postnatal day 60 , two female offspring rats from the same biological mother were selected randomly.

\subsection{Administration of FA in Rats}

FA (purity $>98 \%$ measured by high-performance liquid chromatography) extracted from Ligusticum chuanxiong, was obtained from Shaanxi Huike Botanical Development Co., Ltd (Xi'an, China). Fluoxetine was purchased from Changzhou Siyao Pharmaceuticals Co., Ltd (Changzhou, China). FA and fluoxetine stock solutions were prepared in water containing $0.5 \% \mathrm{CMC}-\mathrm{Na}(\mathrm{m} / \mathrm{v})$. We used a dose-response method to administer, FA at low $12.5 \mathrm{mg} / \mathrm{kg} /$ day (FA-L; $\mathrm{n}=8$ ), medium $25 \mathrm{mg} / \mathrm{kg} /$ day (FA-M; $n=8$ ), high $50 \mathrm{mg} / \mathrm{kg} /$ day (FA-H; $n=8)$. The, male, prenatally-stressed offspring were treated with $0.5 \% \mathrm{CMC}-\mathrm{Na}$, and used as the control group. The PS-fluoxetine group was treated with $20 \mathrm{mg} / \mathrm{kg} /$ day fluoxetine $(n=8)$. All treatments were administered by the intragastric (i.g.) for 4 weeks.

After 4 weeks of FA and fluoxetine administration, we assessed depressive-like behavior in prenatally-stressed offspring rats using the sucrose preference, forced swimming, and open field tests for 6 days. Following behavioral testing, we removed the hippocampus of all groups. Tissue was stored at $-80^{\circ} \mathrm{C}$ until processing for ELISA, mRNA extraction or western blotting. The experimental timeline of this study is shown in Figure 1A.

\subsection{Sucrose Preference Test}

Sucrose preference was assessed using a modified version of the sucrose preference test [35] in male, prenatally-stressed offspring rats. The sucrose consumption tests were performed using a two bottles test, with rats having free access to both water and a sucrose solution. Animals were first trained to consume water in the two bottles, and water consumption was measured for $24 \mathrm{~h}$. The next day, sucrose consumption tests began: a bottle filled with a $2 \%$ sucrose solution replaced a bottle of water for $4 \mathrm{~h}$. Bottles were counterbalanced across the left and the right sides of the feeding compartment, and alternated in position from test to test. Sucrose preference was calculated as follows: sucrose preference $(\%)=$ [sucrose solution intake $(\mathrm{mL}) /$ total fluid intake $(\mathrm{mL})] \times 100$.

\subsection{Forced Swimming Test (FST)}

We used the FST to assess depressive-like behavior in the male prenatal stressed offspring rats. The FST was conducted according to previous reports [34]. Rats were forced to swim in a cylindrical tank (height, $50 \mathrm{~cm}$; diameter, $20 \mathrm{~cm}$ ) filled with water $\left(23-25^{\circ} \mathrm{C}\right)$ to a depth of $40 \mathrm{~cm}$ for $8 \mathrm{~min}$. Movements were recorded during the 8-min session by video camera. Immobility time (measured as floating without active forepaws movements) was recorded manually by a trained rater blinded to the status of each rat. Immobility time was calculated as follows: immobility time $(\%)=[$ immobility time $(\mathrm{s}) /$ total time $(\mathrm{s})] \times 100$. The water in the tank was changed between each test.

\subsection{Open Field Test (OFT)}

We used OFT to assess in the male prenatal stressed offspring. During a 5-min observation period, rats were exposed to an open field $(150 \times 150 \times 49.5 \mathrm{~cm}$; black acrylic walls, green floor), which was divided into a $5 \times 5$ grid of equally-sized squares using white tape. The central region of the box $(3 \times 3)$ was subdivided into a large and a small center of 8 and 1 squares, respectively. The test began by placing each rat on the same side of the small center. Time spent in the center, and the frequency of total crossing counts (total number of squares crossed with all four paws), center crossing counts (total number of center squares crossed with all four paws), rearing counts (standing on hind legs, with or without contact with the sides of the arena), grooming counts (using paws or tongue to clean/scratch body) were recorded. The open field was cleaned with $5 \%$ ethanol between each test. 


\subsection{Enzyme-Linked Immunosorbent Assay (ELISA)}

After behavioral testing, rats were anaesthetized, then decapitated, and the whole brains were removed quickly. The hippocampus was isolated quickly, then frozen in liquid nitrogen and stored at $-80^{\circ} \mathrm{C}$ for posterior analysis. The concentration of IL-10, TNF- $\alpha$, IL- 6 and IL- $1 \beta$ in the hippocampus were measured using a paired antibody quantitative ELISA kit according to manufacturer's instructions (KeyGen BioTECH, Nanjing, China).

Meanwhile, blood was collected from the abdominal aorta for corticosterone detection. The blood was centrifuged $(3500 \times \mathrm{g}, 15 \mathrm{~min})$ at $4{ }^{\circ} \mathrm{C}$, and the serum was stored at $-80^{\circ} \mathrm{C}$ until being used for $\mathrm{ACTH}$ and corticosterone assays. Corticosterone levels were measured by ELISA according to the instructions of the manufacturer (Shanghai Jianglai Biotech, China).

\subsection{RNA Isolation and Real-time PCR}

Real time RT-PCR was used to detect mRNAs for $I L-1 \beta, I L-6, I L-10$ and TNF- $\alpha$. Total mRNA was isolated from tissues using the Trizol method and quantified by Nano-drop spectrophotometry (Thermo Scientific, MA, America). First strand cDNA was synthesized from $1 \mu \mathrm{g}$ of each mRNA sample with random primers and a reverse transcription kit (Takara, Kusatsu, Japan). Primer sequences for $I L-1 \beta, I L-6, I L-10, T N F-\alpha$, and $\beta$-actinmRNA are described in Table 1. SYBR Green real-time PCR Master Mix (Takara, Kusatsu, Japan) was used to detect the abundance of PCR products in the samples. Amplification conditions were: denaturation of all pairs of primers at $94{ }^{\circ} \mathrm{C}$ for $30 \mathrm{~s}$; annealing: $I L-1 \beta$, $60{ }^{\circ} \mathrm{C}$ for $30 \mathrm{~s}, I L-6, I L-10$ and $T N F-\alpha, 56^{\circ} \mathrm{C}$ for $30 \mathrm{~s}$; elongation: $I L-1 \beta, I L-6, I L-10$ and $T N F-\alpha, 72{ }^{\circ} \mathrm{C}$ for $20 \mathrm{~s}$. Forty cycles were performed. The relative expression of $I L-1 \beta, I L-6, I L-10$ and TNF- $\alpha$ mRNA was calculated using the $2^{-\Delta(\Delta C T)}$ comparative method [36], IL-1 $\beta, I L-6$ and TNF- $\alpha$ samples were normalized against the internal endogenous reference, $\beta$-actin.

Table 1. Primers used for IL-1 $\beta$, IL-6, IL-10, TNF- $\alpha$ and GAPDH.

\begin{tabular}{ccc}
\hline Primers & Forward/ Reverse & Sequence \\
\hline \multirow{2}{*}{ IL-1 $\beta$} & Forward & $5^{\prime}$-aatgctcgtgctgctg-3 \\
& Reverse & $5^{\prime}$-tgtcgttgcttgtctctcc- $3^{\prime}$ \\
IL-6 & Forward & $5^{\prime}$-ccagagtcattcagagcaatac-3' \\
& Reverse & $5^{\prime}$-gatggtcttggtccttagcc- $3^{\prime}$ \\
IL-10 & Forward & $5^{\prime}$-tgccttcagtcaagtgaagact- $3^{\prime}$ \\
& Reverse & $5^{\prime}$-aaactcattcatgcttgta-3 \\
TNF- $\alpha$ & Forward & $5^{\prime}$-ccacgctcttctgtctactg-3 \\
& Reverse & $5^{\prime}$-ctacgggcttgtcactcg-3' \\
$\beta$-actin & Forward & $5^{\prime}$-tacaaccttcttgcagctctc-3 \\
& Reverse & $5^{\prime}$-gccgtgttcaatggggtact-3' \\
\hline
\end{tabular}

\subsection{Nissl Staining}

Animals were sacrificed by cardiac perfusion with $4 \%$ paraforaldehyde under anesthesia. The whole brains were removed and processed in paraffin, sectioned at a thickness of $4 \mu \mathrm{m}$, and stained with toluidine blue, which are referred to the previous study [10]. For quantitative studies, neurons in the area of CA3 regions were counted using five equally spaced coronal sections passing through the hippocampus for each brain, and quantified by Image-J software.

\subsection{Immunohistochemical Assay for nNOS}

Animals were euthanized and transcardially perfused with $4 \%$ paraformaldehyde. The brains were removed, post-fixed in $4 \%$ paraformaldehyde for $24 \mathrm{~h}$. The brains were embedded into paraffin and sectioned at a thickness of $4 \mu \mathrm{m}$ (Leica RM2235, Solms, Germany). Slices were washed with PBS for $5 \mathrm{~min} \times 3$ times, then treated with $3 \% \mathrm{H}_{2} \mathrm{O}_{2}$ for $10 \mathrm{~min}$. Sections were washed with PBS for $5 \mathrm{~min}$ $\times 3$ times, and blocked with 5\% BSA for $30 \mathrm{~min}$ and incubated in anti-nNOS (1:500) primary antibody 
diluted in $5 \% \mathrm{BSA}$ overnight at $4{ }^{\circ} \mathrm{C}$, then further incubated with rat anti-rabbit $\operatorname{IgG}\left(30 \mathrm{~min}, 37^{\circ} \mathrm{C}\right)$. After staining with Mayer' hematoxylin for nucleus, sections were mounted on poly-L-lysine-coated glass slides, dehydrated, and covered with coverslips by permount tm mounting medium, which can be referred to previous studies [10]. Imaging $(400 \times)$ was performed using a Nikon Ti-U camera connected to a Nikon Eclipse Timicroscope. The quantified integrated optical density (IOD) of positive nNOS neuron of CA1 region was quantified by Image-J software.

\subsection{Western Blot Analysis}

The protein expression analysis was performed using antibodies against phosphor-NF- $\mathrm{kB}$, GR, IL-1 $\beta$, IL-6, IL-10 and TNF- $\alpha$, as described previously [37]. Total hippocampal proteins were electrophoresed using $10 \%$ tris-glycine sodium dodecyl sulfate polyacrylamide gels. Then, the gels were transferred to PVDF membranes and blocked using 5\% skim milk powder in T-TBS $(0.02 \mathrm{M}$ tris $/ 0.15 \mathrm{M} \mathrm{NaCl}$, pH 7.5, and containing $0.1 \%$ tween 20) at room temperature for $2 \mathrm{~h}$. After washing three times with T-TBS, the PVDF membranes were incubated with primary antibodies against phosphor-NF-KB and GR (Cell Signaling Technology, MA America), IL-1 $\beta$, IL-6, IL-10 and TNF- $\alpha$, (ABclonal, Wuhan, China)overnight at $4{ }^{\circ} \mathrm{C}$. Thereafter, the blots were washed three times with T-TBS and incubated with secondary antibodies (1:4000) at $37^{\circ} \mathrm{C}$ for $2 \mathrm{~h}$. $\beta$-actin was used as an internal control. Finally, after washing three times with T-TBS, the PVDF membranes were developed using enhanced chemiluminescent (ECL) reagent. The intensity of the bands was analyzed with Image-J software. Intensity values of phosphor-NF- $\mathrm{kB}$ were normalized to NF- $\mathrm{kB}$, and GR were normalized to $\beta$-actin.

\subsection{Statistical Analysis}

All data are provided as mean \pm SEM. Statistical analyses were performed by one-way analysis of variance (ANOVA) comparison using SPSS Version 24.0 (Chicago, IL, USA). $p<0.05$ was considered statistically significant.

Author Contributions: Conceptualization, X.Z., Y.C. (Ying Cheng), Y.C. (Yiwei Chen), Y.Y. and Y.C. (Yanjun Cao); Formal analysis, S.Z.X. and J.Z.; Funding acquisition, Y.C. (Yanjun Cao); Methodology, X.Z.; Writing-original draft, X.Z., Y.C. (Ying Cheng), Y.L. (Yingchun Li), Y.L. (Yang Li) and H.D.; Writing-review \& editing, Y.C. (Yanjun Cao).

Funding: This work was supported by National Natural Science Foundation of China (Grant No. 31600822 and 81773870) and Natural Science Foundation of Shaanxi Province (Grant No. 15JS107, 2016JQ8059 and 14NW14).

Conflicts of Interest: The authors declare no conflict of interest.

\section{References}

1. Rice, F.I.; Thapar, J.A. The impact of gestational stress and prenatal growth on emotional problems in offspring: A review. Acta Psychiatr. Scand. 2007, 115, 171-183. [CrossRef] [PubMed]

2. Nogueira, P.J.; Ferreira, H.H.A.; Antunes, E.; Teixeira, N.A. Chronic mild prenatal stress exacerbates the allergen-induced airway inflammation in rats. Mediat. Inflamm. 1999, 8, 119-122. [CrossRef] [PubMed]

3. Wang, Y.; Ma, Y.; Hu, J.; Cheng, W.; Jiang, H.; Zhang, X.; Li, M.; Ren, J.; Li, X. Prenatal chronic mild stress induces depression-like behavior and sex-specific changes in regional glutamate receptor expression patterns in adult rats. Neuroscience 2015, 301, 363-374. [CrossRef] [PubMed]

4. Weinstock, M. The long-term behavioural consequences of prenatal stress. Neurosci. Biobehav. Rev. 2008, 32, 1073-1086. [CrossRef] [PubMed]

5. Mailliot, V.; Wolowczuk, I.; Mairesse, J.; Viltart, O.; Delacre, M.; Khalife, J.; Chartier Harlin, M.C.; Maccari, S. Prenatal stress has pro-inflammatory consequences on the immune system in adult rats. Psychoneuroendocrino 2007, 32, 114-124.

6. Wu, Y. Impact of prenatal stress and adulthood stress on immune system a review. Biomed. Res. 2012, 23, 315-320. 
7. Diz-Chaves, Y.; Astiz, M.; Bellini, MJ.; Garcia-Segura, L.M. Prenatal stress increases the expression of proinflammatory cytokines and exacerbates the inflammatory response to LPS in the hippocampal formation of adult male mice. Brain Behav. Immun. 2013, 28, 196-206. [PubMed]

8. Veru, F.; Laplante, D.P.; Luheshi, G.; King, S. Prenatal maternal stress exposure and immune function in the offspring. Stress 2014, 17, 133-148. [CrossRef]

9. Hankin, B.L. Adolescent depression: Description, causes, and interventions. Epilepsy Behav. 2006, 8, $102-114$.

10. Zhu, Z.L.; Li, X.; Chen, W.N.; Zhao, Y.; Li, H.; Cai, Q.; Jia, N.; Bai, Z.L.; Liu, J.K. Prenatal stress causes gender-dependent neuronal loss and oxidative in Rat Hippocampus. J. Neurosci. Res. 2004, 78, 837-844. [CrossRef]

11. Glover, V.; O'Connor, T.G.; O'Donnell, K. Prenatal stress and the programming of the HPA axis. Neurosci. Biobehav. Rev. 2010, 35, 17-22. [CrossRef] [PubMed]

12. Hodes, G.E.; Kana, V.; Menard, C.; Merad, M.; Russo, S.J. Neuroimmune mechanisms of depression. Nat. Neurosci. 2015, 18, 1386-1393. [CrossRef] [PubMed]

13. Dowlati, Y.; Herrmann, N.; Swardfager, W.; Liu, H.; Sham, L.; Reim, E.K.; Lanctôt, K.L. A Meta-Analysis of Cytokines in Major Depression. Biol. Psychiatry 2010, 67, 446-457. [CrossRef] [PubMed]

14. Dantzer, R.; O'Connor, J.C.; Freund, G.G.; Johnson, R.W.; Kelley, K.W. From inflammation to sickness and depression: When the immune system subjugates the brain. Neuroscience 2008, 9, 47-57. [CrossRef] [PubMed]

15. Kaufmann, F.N.; Costa, A.P.; Ghisleni, G.; Diaz, A.P.; Rodrigues, A.L.S.; Peluffo, H.; Kaster, M.P. NLRP3 inflammasome-driven pathways in depression: Clinical and preclinical findings. Brain Behav. Immun. 2017, 64, 367-383. [CrossRef] [PubMed]

16. Li, H.; Lin, S.; Qin, T.; Li, H.; Ma, Z.; Ma, S. Senegenin exerts anti-depression effect in mice induced by chronic un-predictable mild stress via inhibition of NF-kappaB regulating NLRP3 signal pathway. Int. Immunopharmacol. 2017, 53, 24-32. [CrossRef] [PubMed]

17. Liu, B.; Xu, C.; Wu, X.; Liu, F.; Du, Y.; Sun, J.; Tao, J.; Dong, J. Icariin exerts an antidepressant effect in an unpredictable chronic mild stress model of depression in rats and is associated with the regulation of hippocampal neuroinflammation. Neuroscience 2015, 294, 193-205. [CrossRef]

18. Song, Y.; Sun, R.; Ji, Z.; Li, X.; Fu, Q.; Ma, S. Perilla aldehyde attenuates CUMS-induced depressive-like behaviors via regulating TXNIP/TRX/NLRP3 pathway in rats. Life Sci. 2018, 206, 117-124. [CrossRef]

19. Cao, Y.J.; Zhang, Y.M.; Qi, J.P.; Liu, R.; Zhang, H.; He, L.C. Ferulic acid inhibits $\mathrm{H}_{2} \mathrm{O}_{2}$-induced oxidative stress and inflammation in rat vascular smooth muscle cells via inhibition of the NADPH oxidase and NF-kappaB pathway. Int. Immunopharmacol. 2015, 28, 1018-1025. [CrossRef]

20. Hassanzadeh, P.; Arbabi, E.; Atyabi, F.; Dinarvand, R. Ferulic acid exhibits antiepileptogenic effect and prevents oxidative stress and cognitive impairment in the kindlingmodel of epilepsy. Life Sci. 2017, 179, 9-14. [CrossRef]

21. Ogle, W.O.; Speisman, R.B.; Ormerod, B.K. Potential of treating age-related depression and cognitive decline with nutraceutical approaches: A mini-review. Gerontology 2013, 59, 23-31. [CrossRef] [PubMed]

22. Mamiya, T.; Kise, M.; Morikawa, K. Ferulic acid attenuated cognitive deficits and increase in carbonyl proteins induced by buthionine-sulfoximine in mice. Neurosci. Lett. 2008, 430, 115-118. [CrossRef] [PubMed]

23. Yabe, T.; Hirahara, H.; Harada, N.; Ito, N.; Ito, N.; Nagai, T.; Sanagi, T.; Yamada, H. Ferulic acid induces neural progenitor cell proliferation in vitro and in vivo. Neuroscience 2010, 165, 515-524. [CrossRef] [PubMed]

24. Pincus-Knackstedt, M.K.; Joachim, R.A.; Blois, S.M.; Douglas, A.J.; Orsal, A.S.; Klapp, B.F.; Wahn, U.; Hamelmann, E.; Arck, P.C. Prenatal Stress Enhances Susceptibility of Murine Adult Offspring toward Airway Inflammation. J. Immunol. 2006, 177, 8484-8492. [CrossRef] [PubMed]

25. Plant, D.T.; Pawlby, S.; Sharp, D.; Zunszain, P.A.; Pariante, C.M. Prenatal maternal depression is associated with offspring inflammation at 25 years: A prospective longitudinal cohort study. Transl. Psychiatry 2016, 6, e936. [CrossRef] [PubMed]

26. Nestler, E.J.; Barrot, M.; DiLeone, R.J.; Eisch, A.J.; Gold, S.J.; Monteggia, L.M. Neurobiology of Depression. Neuron 2002, 34, 13-25. [CrossRef]

27. Pittenger, C.; Duman, R.S. Stress, depression, and neuroplasticity: A convergence of mechanisms. Neuropsychopharmacol 2008, 33, 88-109. [CrossRef]

28. Silberman, D.M.; Acosta, G.B.; Zorrilla Zubilete, M.A. Long-term effects of early life stress exposure: Role of epigenetic mechanisms. Pharmacol. Res. 2016, 109, 64-73. [CrossRef] 
29. InbredHarris, A.; Seckl, J. Glucocorticoids, prenatal stress and the programming of disease. Horm. Behav. 2011, 59, 279-289.

30. Anderson, G. Linking the biological underpinnings of depression: Role of mitochondria interactions with melatonin, inflammation, sirtuins, tryptophan catabolites, DNA repair and oxidative and nitrosative stress, with consequences for classification and cognition. Prog. Neuro-Psychopharmacol. 2017, 8, 255-266. [CrossRef]

31. Zeni, A.L.B.; Zomkowski, A.D.E.; Maraschin, M.; Rodrigues, A.L.S.; Tasca, C.I. Ferulic acid exerts antidepressant-like effect in the tail suspension test in mice: Evidence for the involvement of the serotonergic system. Eur. J. Pharmacol. 2012, 679, 68-74. [CrossRef] [PubMed]

32. Zeni, A.L.B.; Zomkowski, A.D.E.; Maraschin, M.; Rodrigues, A.L.S.; Tasca, C. Involvement of PKA, CaMKII, PKC, MAPK/ERK and PI3K in the acute antidepressant-like effect of ferulic acid in the tail suspension test. Pharmacol. Biochem. Behav. 2012, 103, 181-186. [CrossRef] [PubMed]

33. Wenk, G.L.; Gramling, K.M.; Wegrzyniak, B.H.; Ronchetti, D.; Maucci, R.S.; Rosi, L.G.; Ongini, E. Attenuation of chronic neuroinflammation by a nitric oxide-releasing derivative of the antioxidant ferulic acid. J. Neurochem. 2004, 89, 484-493. [CrossRef] [PubMed]

34. Zhang, X.H.; Jia, N.; Zhao, X.Y.; Tang, G.K.; Guan, L.X.; Wang, D.; Sun, H.L.; Li, H.; Zhu, Z.L. Involvement of pGluR1, EAAT2 and EAAT3 in offspring depression induced by prenatal stress. Neuroscience 2013, 250, 333-341. [CrossRef] [PubMed]

35. Sun, H.L.; Guan, L.X.; Zhu, Z.L.; Li, H. Reduced levels of NR1 and NR2A with depression-like behavior in different brain regions in prenatally stressed juvenile offspring. PLoS ONE 2013, 8, e81775. [CrossRef] [PubMed]

36. Shatillo, A.; Salo, R.A.; Giniatullin, R.; Gröhna, O.H. 2015. Involvement of NMDA receptor subtypes in cortical spreading depression in rats assessed by fMRI. Neuropharmacology 2013, 93, 164-170. [CrossRef] [PubMed]

37. Cao, Y.J.; He, X.; Wang, N.; He, L.C. Effects of imperatorin, the active component from Radix Angelicae (Baizhi), on the blood pressure and oxidative stress in 2K,1C hypertensive rats. Phytomedicine 2013, 20, 1048-1054. [CrossRef] [PubMed] 